\title{
A new marker in preterm labor: RDW and MPV
}

\author{
Abstract \\ Aim: To evaluate whether serum platelets Mean Platelet Volum (MPV), and Red Cell \\ Distribution width (RDW) are useful as predictors of preterm labour (PL) in patients with \\ preterm labour (PL), and to compare the clinical efficacy of various serum inflammatory \\ markers to predict the risk of preterm delivery (PD).
}

Materials, and methods: The main group consisted of pregnant women who were earlier than the 37 th gestational week. The control group has consisted of pregnant bigger than 37th weeks of gestation. The patients with PL who participated in the study group were divided into early preterm labour (EPL), and late preterm labour (LPL) groups. The two groups were investigated in terms of clinical aspects of RDW, and MPV and serum markers studied at admission. ROC curve analysis was used to determine the optimal MPV, RDW cut-off levels predicting PL.

Results: Neutrophil (NEU), MPW, RDW, and neutrophil to lymphocyte ratio (NLR) were significantly higher in LPL than in women who gave birth at term $(\mathrm{p}=0.006, \mathrm{OR}=1.411$; $\mathrm{p}<0.001, \mathrm{OR}=1.410 ; 0.002, \mathrm{OR}=1.612, \mathrm{p}=0.035, \mathrm{OR}=1.294)$. In multivariate regression analysis, MPV positive was the strongest predictor variable. Besides, there was a significant correlation between MPV and RDW elevation, and neonatal intensive care needs (NICU) in women who delivered between 34 weeks, and 37 weeks.

Conclusion: High RDW and MPV are independent predictors of preterm delivery in patients with LPL. In our study, we found that the increase of RDW and MPV was higher in women with high PL risk more than in healthy individuals. MPV has the highest area for prediction of preterm birth, and $\mathrm{RDW}>14,5$, and $\mathrm{MPV}>9,6$ have the highest sensitivity and specificity. RDW may be more significant than measuring any of the individual markers in the simultaneous use of preterm delivery. The simultaneous use of RDW and MPV with existing markers to increase our identifying abilities of preterm labour may be stronger than that of any of the individual markers.

Keywords: preterm labour, red cell distribution, mean platelet volum, neutrophil to lymphocyte ratio
Volume II Issue 6 - 2020

\author{
Yüksel Kurban, ${ }^{3}$ Yasemin Alan, ${ }^{2}$ Murat Alan, ${ }^{3}$ \\ Mustafa Kurt, ${ }^{4}$ Beril Gurlek, ${ }^{5}$ Onur Burak \\ Cegilli, ${ }^{3}$ Cüneyt Eftal Taner, ${ }^{3}$ Abdulmecit \\ Öktem ${ }^{3}$ \\ 'Department Obstetrics and Gynecology, Yuksek Ihtisas \\ University, Ankara Medicalpark Hospital, Turkey \\ ${ }^{2}$ Department Obstetrics and Gynecology Metropolitan \\ Municipality Esrefpasa Hospital Clinic Izmir,Turkey \\ ${ }^{3}$ Department of Obstetrics and Gynecology, University of \\ Health Sciences Tepecik Education and Research Hospital, İzmir, \\ Turkey \\ ${ }^{4}$ Department of Obstetrics and Gynecology, Hitit University, \\ Turkey \\ ${ }^{5}$ Department of Obstetrics and Gynecology, Recep Tayyip \\ Erdogan University, Turkey
}

Correspondence: MD.Yüksel Kurban, Department Obstetrics, and Gynecology, Yuksek Ihtisas University, Ankara Medicalpark Hospital,Ankara, Kent Koop Mh. 06100. Batıkent, Yenimahalle, Ankara, Turkey, Tel 905326872263 , Email drykurban@hotmail.com

Received: November 25, 2020 | Published: December 17, 2020
Abbreviations: MPV, mean platelet volum; RDW, red cell distribution width; PL, preterm labour; PD, preterm delivery; EPL, early preterm labour; LPL, late preterm labour; NEU, neutrophil; NLR, neutrophil to lymphocyte ratio; NICU, neonatal intensive care needs; CRP, C-reactive protein C; WBC, white blood cell; PLT, platelet; GDM, gestational diabetes mellitus; LYN, lymphocyte; EDTA, ethylenediaminetetraacetic acid; AUC, area under the curve; IL, interleukin; TNF alpha, tumour necrosis factor-alpha; IGFBP-1, growth factor-binding protein-1; $\mathrm{RBC}$, red blood cell; RDS, respiratory distress syndrome

\section{Introduction}

Delivery before 37 weeks of gestation affects $5 \%$ to $18 \%$ of all pregnancies, while it is also a major cause of perinatal adverse outcomes. ${ }^{1}$ Despite the intense development of early diagnosis and rapid treatment in recent years, the rate of $\mathrm{PB}$ continues to increase worldwide. Nearly 1.1 million babies die each year because of the prematurity. The survivors face both short, and long-term sequelae. ${ }^{2}$ In PD, higher mortality-related problems usually developed in the weeks before the 34th week, but more than $65 \%$ of PDs occur between the 34 th, and 37 th gestational weeks (in the late preterm period). ${ }^{3}$ To avoid inadequate interventions, the mechanisms, and the causes of preterm labour in high-risk pregnant women should be investigated. Since PB is associated with several complications, it is very important to diagnose the early treatment of inflammatory response or oxidative stress that is responsible for the pathogenesis to identify strategies for treatment, and thus to improve prognosis. ${ }^{4}$ Intrauterine inflammation produces a maternal inflammatory response leading to infiltration into the chorion, amnion, or decidua, while the inflammatory response results in inflammation extending to the chorionic plaque, umbilical cord, and fetüs. ${ }^{5}$ Prenatal diagnoses are especially important because of intrauterine infection, and/or inflammation, which is the main component of PL, is associated with poor prognosis, because preterm infants survival can also increase the risk of long-term poor prognosis. ${ }^{4,5}$ However, there is still no adequate diagnostic tool for early prenatal diagnosis of PL. Cervical length measurement, amniotic fluid culture, C-reactive protein $\mathrm{C}(\mathrm{CRP})$, cytokine levels, cervicovaginal fetal fibronectin test in maternal serum are the recommended methods for EPL prediction. ${ }^{6-8}$

Recently, many haematological markers such as white blood cell (WBC) subtypes, and platelet (PLT) have been mentioned as diagnostic, and prognostic factors in a certain type of diseases, including obstetric diseases. Although many studies focused on intrauterine infection, and inflammation markers to find PB-sensitive, less invasive methods, there is still no effective, inexpensive, and easily accessible marker. ${ }^{5}$

RDW is a measure of discrepancy in the magnitude of circulating erythrocytes that reflect variability in cell sizes. RDW has been used primarily in the differential diagnosis of microcytic anaemia but has recently been proposed as a predictive, and prognostic parameter in different pathologies in adult patients. RDW increases in the event of ineffective production or increased damage to red blood cells in 
inflammatory or infectious conditions. ${ }^{9,10}$ MPV is a reliable PLT size indicator that reflects PLT function, and activation. The increase in MPV was attributed to the production of young, and large PLTs by consuming PLTs in inflammation. Large PLTs have denser granules than small PLTs, and are more metabolically, and enzymatically active, and have a higher metabolism. So far, studies on MPV in LPL patients are inadequate. ${ }^{11,12}$ Therefore, considering the fact that subclinical intrauterine infection is one of the most frequently responsible pathologies in the preterm labour mechanism, investigating the importance of this marker in LPL will contribute to the literature.

Recent studies in pregnant women have shown blood markers that reflect systemic inflammation are likely independent diagnostic, and prognostic factors of gestational diabetes mellitus (GDM), and many subclinical inflammatory diseases including preeclampsia, and PL., ${ }^{9,1}$ However, there is little data in the literature on whether these systemic inflammatory biomarkers are associated with PL. In our study, it is aimed to investigate the role of MPV, and RDW in predicting PL, and whether these two markers can be used as appropriate parameters to estimate PL severity and NICU risk.

\section{Materials and methods}

Patients between 18-34years of age in our hospital were examined within three years between January 2015, and December 2017. The Local Ethics Committee approved the study. The universal principles of the Helsinki Declaration were implemented. We used the perinatology department's database to find out pregnancies with PL. In the sterile speculum, and digital examination of patients between $24(0 / 7)$, and $36(6 / 7)$ weeks of gestation, cervical effacement $\geq 80 \%$, and $1-3 \mathrm{~cm}$ cervical dilatation or spontaneous presence of persistent uterine contractions (at least four contractions within twenty minutes, and contractions lasting 30 seconds or longer) was considered PL. PL was defined as LPL (34-37 weeks), and EPL ( $<34$ weeks). A term birth is the birth of a fetus at $37-42$ when the pregnancy is complete.

At first, the obstetric recordings of all patients with PL diagnosis were examined. When multiple applications were made for PL, the gestational week at the time of admission was recorded for analysis. Although the cervical length measurement and obstetric ultrasonography were performed at another centre in patients presenting with the diagnosis of PL, and intact membranes, these measurements were made, and recorded in all patients in a st, the standardized technique. ${ }^{13}$ Since our primary interest is the time interval between labour, and delivery, records of pregnancies that performed $\mathrm{PB}$ in the first hospitalization or who gave birth while under observation, and treatment in the hospital were also collected. During this period, pregnancies lost or not reached during follow-up were excluded. In addition to interviews with patients, gravida, body mass index, the form of conception (in vitro fertilization, spontaneous), parity, fetal gender, PD history, gestational age at admission, and delivery, type of delivery, APGAR score, NICU the requirement, neutrophil (NEU), lymphocyte (LYN), NLR, MPV, and RDW data are recorded.

According to our protocol, ultrasonography, and vaginal sterile speculum examination were performed to all patients with hospitalized symptoms for post-diagnostic surveillance, and digitally examined for dilatation, and effacement evaluation. Complete blood count was obtained from all patients before antibiotic, corticosteroid or tocolytic treatment. Conservative treatment is recommended in patients with no fetal or maternal indications for delivery. Fetal well-being and uterine contractions were observed, and cervical changes were discontinued. Patients whose pain was continued were monitored spontaneously. Patients that did not develop preterm labour were discharged. Last menstrual period and ultrasonographic screening in the first or second trimester are used to calculate the gestational age. We evaluated and described the pregnancy outcome from the time of admission to delivery.

Body mass index (BMI) $\left(\geq 30 \mathrm{~kg} / \mathrm{m}^{2}\right)$, multiple gestations, history of PL in a previous pregnancy, history of cervical cerclage, and amniocentesis in the current pregnancy, oligohydramnios, polyhydramnios, smoking, stillbirth, fetus complicated by chromosomal anomalies, steroid application, preterm early membrane rupture (PPROM), malignancy, GDM (Gestational diabetes mellitus), any acute or chronic infectious or inflammatory disease, sepsis, Hemoglobulin $<10$, thrombocytopenia (PLT less than 100 000), thrombocytosis (PLT greater than 500 000), uterine anomalies, preeclampsia, hypertension, coagulation disorders, deep vein thrombosis, those who use drugs that affect PLT functions such as acetylsalicylic acid were excluded from the study. Patients with significant obstetric or medical complications, and non-reassuring maternal, and fetal status information, clinical chorioamnionitis, placental abruption, antepartum haemorrhage, and intrauterine growth restriction (estimated st, standard birth weightless than $10 \%$ below the age required for gestational age) where not included in the study. The presence of maternal fever $(>38 \mathrm{C})$ with two of the following symptoms in the absence of other potential infectious sources are defined clinic chorioamnionitis: maternal tachycardia $(>100 \mathrm{BPM})$, maternal leukocytosis $\left(15,000\right.$ cells $\left./ \mathrm{mm}^{3}\right)$, uterus sensitivity, fetal tachycardia (>160BPM), and bad smell of amniotic fluid. Patients with PL were divided into two groups according to the period between labour, and delivery. Patients delivered between 34, and 37 weeks were considered as a late preterm delivery group, 37 weeks or more is tagged as a term delivery group. Clinical features and serum markers obtained at admission were used at the comparison of these groups. Subtypes of leukocyte, erythrocyte, platelet counts were assayed with EDTA plasma (Coulter LH 750 analyzer; Beckman Coulter, Galway, Irel, and). Tubes incapsulating ethylenediaminetetraacetic acid (EDTA) were used for after fasting venous blood samples. Samples stored at room temperature for 2 hours, all samples were run in the same centre.

\section{Statistical methods}

SPSS (Version 22.0, SPSS Inc., Chicago, IL, USA) was used to analyze the data collected. Descriptive statistics were presented as the mean \pm st, standard deviation for normal distributed numerical data, median (min-max) for non-normal distributed numerical data, number, and percentage for categorical data. Normality was assessed by the Kolmogorov-Smirnov test. Before the statistical analysis, a matching control based on the propensity score was used to create a control group similar to the age, and BMI data distribution of the LPL group. The groups were similar in terms of confound covariates. In the continuous variables we obtained in our study, we used the Student's t-test when the parametric test assumptions were provided for the independent two numerical mean comparisons. The Mann Whitney U test was used when parametric test assumptions were not provided. The ratio comparison of categorical variables was done by the Chi-square test. In the estimation of LPL, it was investigated statistically whether the parameters of NEU, LYM, MPV, RDW, NLR, WBC were diagnostic and prognostic markers. ROC (Receiver Operating Characteristic) graphs were plotted for determining the discrimination of the parameters (maximum sensitivity and selectivity), the area under the curve (AUC), and the $95 \%$ confidence intervals of this area were calculated. In the analyzes, the significance 
of determining the risk group was evaluated as 0.9-1: Excellent, 0.80.9: good, 0.7-0.8: moderate, 0.6-0.7: weak, and0.5-0.6: failure for AUC. Sensitivity, selectivity, positive predictive value, negative predictive value, and positive likelihood values were calculated for those who were successful according to AUC (AUC $>0.6$ ). You den index was used to determine the best cut-off point in ROC analysis. To determine the effect of the parameters before the ROC analysis in late preterm, univariate, and multivariate binary logistic regression analysis was applied by using the backward Wald method. The relationship between MPV and time from the onset of labour to delivery was analysed with the Kaplan-Meier method. The log-rank test was used to calculate the difference between survival curves. $\mathrm{P}<0.05$ was considered to be statistically significant.

\section{Results}

In the study, the mean age of the group $(n=521)$ was $24.61 \pm 4.46$ BMI mean 25.43 \pm 2.62 years, LPL group $(n=521)$ mean age was $24.46 \pm 4.48$, and the mean BMI was $25.59 \pm 2.61$. Groups were similar in terms of age, and BMI ( $\mathrm{p}=0.584, \mathrm{p}=0.343$, respectively). Clinical features of patients were presented in Table 1.

Comparison of serum marker levels according to groups was given in Table 2. The NEU, LYM, MPV, RDW, NLR, WBC parameters were significantly varied between groups $(\mathrm{p}<0.001,0.047,<0.001$, $<0.001,0.010,<0.001$, respectively (Table 2).

As a result of univariatebinary logistic regression analysis to determine the effect ofthe parameters on LPL, all parameters were found to be significant on LPL ( $<<0.001,0.008,<0.001,<0.001,0.026$, 0.004 , respectively (Table 3 ). Multivariate binary logistic regression analysis revealed that NEU, MPV, RDW, andNLR had a significant effect for LPL in the multimodal model $(\mathrm{p}=0.006, \mathrm{OR}=1.411 ; \mathrm{p}<0.001$, $\mathrm{OR}=1.410 ; \mathrm{p}=0.002, \mathrm{OR}=1.612 . \mathrm{p}=0.035, \mathrm{OR}=1.294$ (Table 3).

Sensitivity, and specificity values for the NEU, MPV, RDW, NLR parameters of the area under the ROC curve (AUC) value of 0.60 were given (Table 4) for the diagnostic capabilities of serum markers. The most successful marker was 0.748 (0.697-0.799) AUC, $0.738(0.698-0.775)$ sensitivity, and $0.712(0.670-0.750)$ MPV with specificity values (cut-off=9.6). The next most successful was RDW (cut-off $=14.5)$. Other values are given (Table 4).

The LPL group was divided into two groups as the patients who needed NICU, and those who don't need NICU, and we compared the MPV, and RDW values in the groups. MPV and RDW values were significantly higher in the NICU group compared to the group without the need for NICU ( $\mathrm{p}=0.002,<0.001$, respectively).

Table I Clinical characteristics of the study population

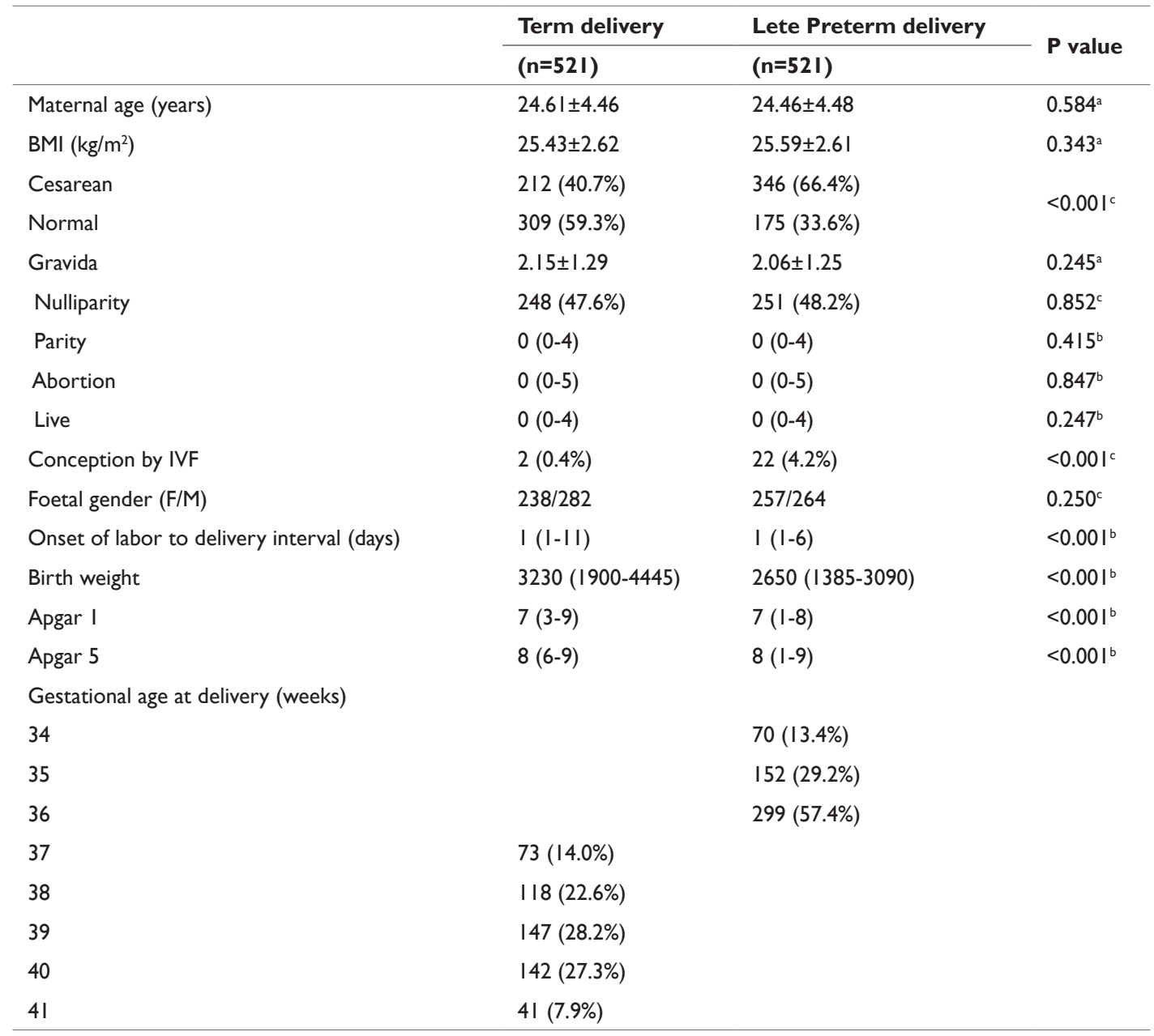

${ }^{a}$ : student's $t$ test, ${ }^{b}:$ mann whitney $U$ test, ${ }^{c}$ : chi-square test

BMI: body mass index; IVF: in vitro fertilisation; F: female; M: male 
Table 2 Mean levels of serum markers in preterm delivery groups

\begin{tabular}{llll}
\hline & Preterm delivery & Term delivery & \multirow{2}{*}{ P value } \\
\cline { 2 - 3 } & $(\mathbf{n}=\mathbf{5 2 1})$ & $(\mathbf{n}=\mathbf{5 2} \mathbf{1})$ & $<0.00 I^{* *}$ \\
\hline NEU & $11.38 \pm 3.74$ & $9.93 \pm 3.12$ & $0.047^{*}$ \\
LYM & $1.84 \pm 0.79$ & $1.94 \pm 0.83$ & $<0.00 I^{* *}$ \\
MPV & $9.56 \pm 1.38$ & $8.21 \pm 1.07$ & $<0.00 I^{* *}$ \\
RDW & $14.75 \pm 2.31$ & $13.83 \pm 2.11$ & $0.010^{*}$ \\
NLR & $7.59 \pm 4.95$ & $6.84 \pm 4.45$ & $<0.00 I^{* *}$ \\
WBC & $13.17 \pm 3.23$ & $12.48 \pm 2.76$ &
\end{tabular}

*Student's $t$ test statistically significant $\left({ }^{*} p<0.05, * * p<0.001\right)$

NLR: neutrophil to lymphocyte ratio

Table 3 Significant predictors of preterm delivery in univariate, and multivariate binary logistic regression analysis

\begin{tabular}{|c|c|c|c|c|c|c|c|c|}
\hline & \multicolumn{4}{|c|}{ Univariate } & \multicolumn{4}{|c|}{ Multivariate } \\
\hline & \multirow{2}{*}{ P value } & \multirow{2}{*}{ OR } & \multicolumn{2}{|c|}{ OR $(95 \% \mathrm{Cl})$} & \multirow{2}{*}{$P$ value } & \multirow{2}{*}{ OR } & \multicolumn{2}{|c|}{ OR $(95 \% \mathrm{Cl})$} \\
\hline & & & Lower & Upper & & & Lower & Upper \\
\hline NEU & $<0.00 I^{*}$ & 1.438 & 1.199 & $\mathrm{I} .774$ & $0.006 *$ & 1.411 & I.I 178 & $1.76 \mathrm{I}$ \\
\hline LYM & $0.008^{*}$ & 0.943 & 0.913 & 0.976 & 0.242 & - & - & - \\
\hline MPV & $<0.00 I^{*}$ & 1.43 & 1.281 & 1.583 & $<0.001 *$ & 1.41 & 1.27 & 1.563 \\
\hline RDW & $<0.00 I^{*}$ & 1.63 & 1.101 & 2.221 & $0.002 *$ & 1.612 & 1.094 & 2.255 \\
\hline NLR & $0.026^{*}$ & 1.343 & 1.205 & 1.59 & $0.035^{*}$ & 1.294 & 1.165 & $1.56 \mid$ \\
\hline WBC & $0.004 *$ & 1.082 & 1.008 & 1.162 & 0.118 & - & - & - \\
\hline
\end{tabular}

*statistically significant $(\mathrm{p}<0.05)$

Table 4 Diagnostic sensitivity, andspecificity of serum markers in study groups

\begin{tabular}{|c|c|c|c|c|c|c|c|}
\hline & AUC & Sensitivity\% & Specificity\% & PPV\% & NPV\% & $L R+[c]$ & Cut-off \\
\hline & $(95 \% \mathrm{Cl})$ & $(95 \% \mathrm{Cl})$ & $(95 \% \mathrm{Cl})$ & $(95 \% \mathrm{Cl})$ & $(95 \% \mathrm{Cl})$ & $(95 \% \mathrm{Cl})$ & value \\
\hline MPV & $\begin{array}{l}0.748 \\
(0.697-0.799)\end{array}$ & $\begin{array}{l}0.738 \\
(0.698-0.775)\end{array}$ & $\begin{array}{l}0.712 \\
(0.670-0.750)\end{array}$ & $\begin{array}{l}0.719 \\
(0.679-0.756)\end{array}$ & $\begin{array}{l}0.731 \\
(0.690-0.769)\end{array}$ & $2.56(2.22-2.96)$ & 9.6 \\
\hline RDW & $\begin{array}{l}0.737 \\
(0.689-0.788)\end{array}$ & $\begin{array}{l}0.71 \\
(0.669-0.748)\end{array}$ & $\begin{array}{l}0.721 \\
(0.680-0.759)\end{array}$ & $\begin{array}{l}0.718 \\
(0.677-0.756)\end{array}$ & $\begin{array}{l}0.713 \\
(0.672-0.75 I)\end{array}$ & $2.55(2.19-2.96)$ & 14.5 \\
\hline NEU & $\begin{array}{l}0.695 \\
(0.654-0.737)\end{array}$ & $\begin{array}{l}0.683 \\
(0.642-0.722)\end{array}$ & $\begin{array}{l}0.631 \\
(0.580-0.679)\end{array}$ & $\begin{array}{l}0.725 \\
(0.684-0.763)\end{array}$ & $\begin{array}{l}0.583 \\
(0.534-0.631)\end{array}$ & $1.85(1.60-2.14)$ & 10.8 \\
\hline NLR & $\begin{array}{l}0.683 \\
(0.643-0.726)\end{array}$ & $\begin{array}{l}0.671 \\
(0.629-0.71 I)\end{array}$ & $\begin{array}{l}0.614 \\
(0.570-0.655)\end{array}$ & $\begin{array}{l}0.635 \\
(0.593-0.675)\end{array}$ & $\begin{array}{l}0.651 \\
(0.607-0.693)\end{array}$ & $1.74(1.53-1.97)$ & 6.9 \\
\hline WBC & $\begin{array}{l}0.595 \\
(0.547-0.643)\end{array}$ & - & - & - & - & - & - \\
\hline LYM & $\begin{array}{l}0.588 \\
(0.539-0.634)\end{array}$ & - & - & - & - & - & - \\
\hline
\end{tabular}


The Kaplan-Meier method revealed that patients with MPV positive (>9.6) had a significantly shorter interval between the onset of labour, and delivery than patients with MPV negative $(\leq 9.6)$ (mean 5.48 versus 8.72 days; log-rank, $\mathrm{p}<0.001$ ) (Figure 1).

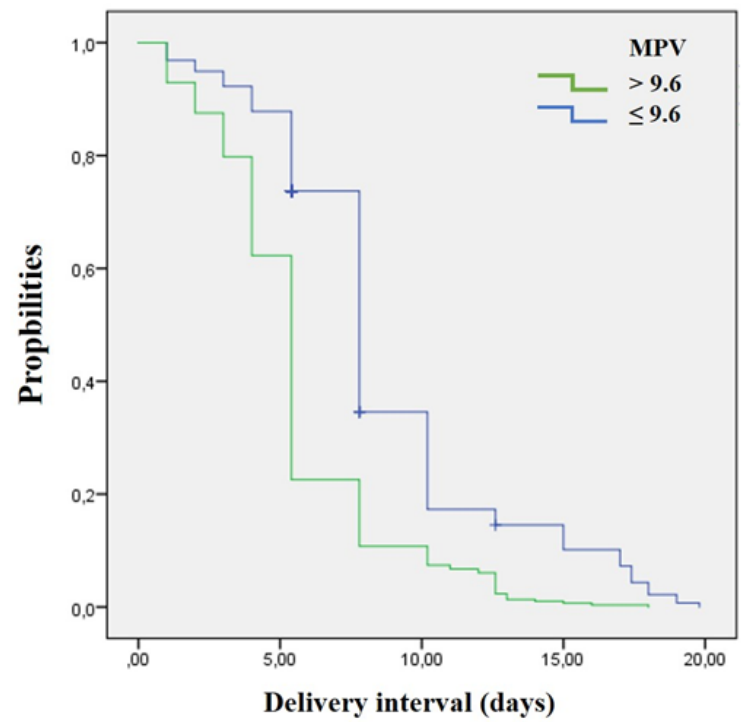

Figure I Kaplan-Meier method of onset of labour to delivery interval according to the optimal cut-off point of MPV ( $\leq 9.6$ versus $>9.85)$.

\section{Discussion}

PD is a crucial motive of neonatal mortality and morbidity. $45 \%$ of preterm births are caused by spontaneous preterm labour. Late preterm births account for approximately $74 \%$ of all PB. $25 \%$, and $30 \%$ of PD are caused by PPROM, and maternal, and fetal infections respectively. ${ }^{2}$ It is known that they have higher mortality, and morbidity compared to term babies in the newborn period. ${ }^{3}$ Possible pathological processes for PB include inflammation, uteroplacental ischemia, immunological reactions, and maternal stress. ${ }^{4,14}$ In this study, we investigated the diagnostic ability of RDW, and MPV to foresee the time of delivery in women with spontaneous LPL.

Since the majority of deaths in PL are $<32$ weeks of gestation, current strategies to predict or prevent the preterm birth is designed for the early preterm group, but it is insufficient data in the literature to determine preterm patients between 34 , and 37 weeks of age. However, it should be kept in mind that newborns born at 34-37 weeks of age have higher mortality and morbidity rates than normal newborns. PL is a multi- etiologic disease including intrauterine infection, and inflammation, genetic factors, cervical disease, uteroplacental ischemia or haemorrhage, endocrine disorders, early activation of the fetal endocrine system, immunological factors. ${ }^{14}$ The vast majority of PL-developing patients are not identifiable, however, it would be ideal for the patient to anticipate that they are at risk for PL, and then to offer preventive interventions.

The measurement of cervical length by transvaginal ultrasonography has been a non-invasive method used to predict preterm delivery. ${ }^{715}$ Transvaginal ultrasound examination and pelvic examination of the majority of women undergoing these procedures may experience discomfort in terms of patient satisfaction in the process of diagnosis, follow-up, and treatment of these processes is problematic. Fetal fibronectin positivity in cervical shortening and cervicovaginal secretions is a valuable method in EPL estimates. However, fetal fibronectin begins to deteriorate naturally after 34 weeks of gestation. ${ }^{16}$ Similarly, cervical length measurement becomes less useful in LPL in predicting preterm labour as the cervix becomes physiologically shorter after 32-34 weeks. ${ }^{15}$ In conclusion, both cervical length measurement, and fetal fibronectin test provide insufficient information about the future of pregnancy in patients with preterm labour after 34 weeks of pregnancy. Although in most cases the pathophysiological mechanism is not clearly defined, a common component of PD is inflammation in the maternal-fetal interface and plays a role in the onset, and subsequent delivery.

The role of this inflammation in PL has been evaluated in many studies, and a significant relationship between PL and various inflammatory markers have been reported. It has been shown that the levels of Interleukin (IL) 1,6, and 8 in amniotic fluid from these inflammatory markers can be used for prediction in patients with PL. ${ }^{17}$

Since infection is chronic, and asymptomatic until the membranes are ruptured, it is still an important problem to identify these invasive procedures in women with PL diagnosis. However, the most reliable method for diagnosing intrauterine infection is amniotic fluid culture from amniotic fluid, but it is unclear that amniocentesis gives better results of women with PL. ${ }^{18}$ Also, amniocentesis is associated with a risk of fetal loss of about $0.5 \%$, and there is a greater need to use a non-invasive method in the diagnosis of PL. There is evidence that proinflammatory cytokines such as serum IL-6, IL-8, tumour necrosis factor-alpha (TNF alpha) contribute to inflammation in PL; however, it is defined as a non-popular preterm marker because of its cost, and inaccessibility. ${ }^{3,4,17} \mathrm{CRP}$, produced as an acute-phase protein, is commonly used in monitoring various inflammatory conditions such as chorioamnionitis in obstetrics practice, and in identifying cases of infection that may increase the risk of PL. However, the potential disadvantage in the evaluation of acute-phase reactants is that they are affected by many conditions, including age, and anaemia other than infection or inflammation. ${ }^{19}$ Despite its importance as a marker of infection, and inflammation, leukocyte counting, and limitation of NLR have a limited diagnostic value for sensitivity and specificity. In addition, the fact that neutrophilia and lymphocytopenia are general innate immune responses to various stressful events of pregnancy, as well as the fact that these markers are strongly influenced by factors such as acute infective or inflammatory processes, physical activity, malnutrition, and drug therapies have led us to focus on the discovery of new markers in the determination of PL. ${ }^{20}$ Recent research shows insulin-like growth factor-binding protein-1 (IGFBP-1) as a potentially promising biomarker. It has been suggested that IGFBP-1 is a better predictor of fetal fibronectin in predicting PB. However, there is little information about sensitivity, specificity, and positive predictive value to determine the clinical usefulness of IGFBP-1, and its cost is problematic. ${ }^{21}$ In general, fetal fibronectin test, amniotic fluid analysis, CRP, and leukocyte levels are important methods for diagnosing inflammation, but studies comparing these markers with each other are available in the literature. While the use of these markers was found to be significant in determining PL in their combined use, it was observed that PL alone could not be predicted independently.

Any disease involving red blood cell (RBC) destruction or production may increase RBC size variability, and lead to RDW elevation. This simple marker has been shown to have a role in predicting adverse outcomes in a variety of clinical conditions including sepsis, coronary artery disease, heart failure, acute pancreatitis, malignancy, and infective endocarditis. ${ }^{9}$ The main mechanism for the increase of RDW in all the diseases mentioned above is not clear. Increased circulating cytokines in inflammation, 
and infection cause irregularities in erythropoiesis. In this case, rising RDW reflects the increased 3 release of inflammatory cytokine levels such as IL-6, TNF-alpha, in inflammation. Another mechanism underlying the increase of RDW is that oxidative stress may affect the erythroid tissue, and circulating erythrocytes. ${ }^{9,10,22}$

RDW of late preterm pregnancies was higher than the full-term pregnancies. Therefore, we can say that an unstable erythropoiesis, and/or stress condition is reflected in high RDW values, especially in preterm pregnancy. It was particularly important in our group selected from non-anemic patients, in which high RDW has demonstrated the underlying inflammatory stress. In our study, we showed that RDW's above a threshold of $14.5 \%$ may be more likely to cause PL. RDW and MPV increase was a significant observation in our study as the time between the first admission to hospital, and delivery time shortened, and RDW and MPV increased in parallel with the severity of PL disease. Özdogan et al. found that there was a positive correlation between RDW, and CRP levels and inflammation caused increased RDW. We found a significant relationship between higher RDW, and NICU in preterm groups. ${ }^{23}$ We can consider the prognostic role of RDW for PL severity, and NICU, both because it is a sample size and a marker that can be easily monitored by a routine $\mathrm{CBC}$ control. Another study on this issue may show our possibilities as supportive.

MPV increases in cases where peripheral platelet (PLT) destruction is increased and decreases in cases where PLT production deteriorates. It causes some morphological changes in PLTs in acute and chronic inflammation. PLTs appear to be larger in shape by globalizing, and forming pseudopodia. ${ }^{11,12}$ As a result, PLTs with increased numbers, and sizes of increased pseudopodia will cause major changes in MPV. A study by Erez et al. suggested that preterm labour was an increased thrombin activation which could result in PLT activation. ${ }^{24}$ Several studies have examined the relationship between MPV, and various diseases. In diabetes, hypercholesterolemia, atherosclerotic renal artery stenosis, ischemic stroke, smokers, deep vein thrombosis, MPV was found to be increased. ${ }^{11,12}$ It has long been known that inflammation is an important stimulus for PLTs and that MPV values increase in low-grade inflammatory disorders. It can be asserted that levels of IL-3, IL-6 from proinflammatory cytokines, which play an important role in PL pathogenesis, may stimulate PLT production, and cause the release of large volumes of PLT from bone marrow. ${ }^{11,12,24}$ In our study, we think that elevating MPV level may be a result of possible low-grade inflammation in PL etiology.

PLT can play a role in the collection of leukocytes by forming a connection bridge between leukocyte (WBC), and endothelium. In a previous study by Saleh et al. mean platelet factor- 4 , and betatromboglobulin plasma concentrations were shown to be higher in the circulation of PL pregnant women. ${ }^{25}$ SCD $40 \mathrm{~L}$ antagonizes the synthesis of NO, which inhibits platelet adhesion, activation, and aggregation, and causes the production of free oxygen radicals that cause endothelial dysfunction, and an increase in inflammatory cytokines. Activated PLTs produce more sCD40L, which further enhances the inflammatory reaction. Consistent with the possible PLT activation in PL, sCD40L levels were higher in preterm labour compared with those who delivered term. ${ }^{26}$

Changes in PLT indices are often associated with PLT consumption, which is the result of an inflammatory process. Gasparyan et al. suggested that MPV, which acts as a prothrombotic or proinflammatory agent, is increasingly used in many diseases, and PLT indices have been reported to be impaired in some obstetric conditions such as recurrent pregnancy loss, preeclampsia, gestational diabetes, and preterm labour. ${ }^{12}$ Ahmed et al. suggested that pregnant women with high MPV in the second, and third trimester are at risk of development of preeclampsia. ${ }^{27}$ In a study performed by Myatt et al., reported that MPV was significantly higher in the first trimester of women who developed preeclampsia. ${ }^{28}$ Aynıoğlu et al. found a higher number of PLT counts, and platelet indices compared to normal pregnancies in recurrent pregnancy losses.$^{29}$ Shahbaz et al. evaluated the relationship of GDM with different PLT indices, and found a statistically significant increase in PLT, MPV, and PDW values according to healthy pregnancies. ${ }^{30}$ Ekin et al. showed that MPV can be used for early diagnosis of PPROM. ${ }^{31}$ Gioia et al. they found that MPV was considerably higher in patients with abnormal umbilical artery Doppler velocimetry and that the MPV value was 10 , and above was significantly associated with adverse neonatal outcomes such as respiratory distress syndrome (RDS), and brain injury. ${ }^{32} \mathrm{We}$ believe that MPV is an important indicator for predicting PL severity, and determining the NICU need, and we believe that this will be reinforced with further studies.

\section{What are our contributions to the literature}

There are a few values in the context of the possible outcome of the present study. Considering the inclusion of a large number of participants enrolled in the present study, and the correlation of NICU requirement with RDW, and MPV, our study is an important addition to the relevant literature. First of all, we can say that we compare a homogeneous situation despite having a retrospective design. When the patients were selected, the distribution was equal in both groups, depending on a previous pairing procedure for patient ages (18-34), and BMI $(<30 \mathrm{~kg} / \mathrm{m})$. We analyzed all samples throughout the entire study period, and with the same instrument for the entire study group. Besides, we excluded several obstetric, and medical conditions that increased the quality of our study, and had the potential to affect the parameters studied. Maternal characteristics and obstetric history are important in the aetiology of PL. These patients were excluded from the study because preterm delivery or PL history was the strongest risk factor. However, these factors are less likely to affect the outcome of the study because pregnant women with moderate or severe anaemia $(\mathrm{Hb}<10 \mathrm{~g} \%)$, platelets less than 50thous, and, and more than 500 thous, and, and blood transfusions are excluded from the study. There were some limitations to be considered in our study. First, RDW, and MPV which are the sign of inflammation may be affected by nutritional status, lifestyle. Furthermore, since we use a single blood sample to calculate MPV and RDW, it is unclear whether a single sample reflects elevated serum markers over time. Second, this study was carried out in a single institution. The results of this study can be highly helpful in the early, and effective treatment of PL. The performance of the multicenter studies of the markers used here will strengthen our results.

\section{Acknowledgments}

The authors thank Mehmet Calan, MD, for data management, and statistical analysis at the Department of Endocrinology Bozyaka Education, andResearch Hospital.

\section{Funding}

This study received no funding.

\section{Conflicts of interest}

There is no conflict of interest in this work. 


\section{References}

1. Hannah B, Simon C, Mikkel ZO, et al. National, regional,, andworldwide estimates of preterm birth rates in the year 2010 with time trends since 1990 for selected countries: a systematic analysis, andimplications. Lancet. 2012;379:2162-2172.

2. Chang HH, Larson J, Belncowe H. Preventing preterm births: analysis of trends, andpotential reductions with interventions in 39 countries with very high human development index. Lancet. 2013;381(9862):223-234.

3. Goldenberg RL, Culhane JF, Iams JD, et al. Epidemiology, andcauses of preterm birth. Lancet. 2008;371:75-84.

4. Ferguson KK, McelrathTF, Chen YH, et al. Repeated measures of urinary oxidative stress biomarkers during pregnancy, andpreterm birth. Am J Obstet Gynecol. 2015;212:1-8.

5. Gotsch F, Gotsch F, Romero R, et al. The preterm parturition syndrome, andits implications for underst, anding the biology, risk assessment, diagnosis, treatment, andprevention of preterm birth. The Journal of Maternal Fetal, and Neonatal Medicine. 2009;22(Suppl 2):5-23.

6. Peaceman AM, Andrews WW, Thorp JM, et al. Fetal fibronectin as a predictor of preterm birth in patients with symptoms: a multicenter trial. American Journal of Obstetrics, and Gynecology. 1997;177:13-18.

7. Sotiriadis A, Papatheodorou S, Kavvadias A, et al. Transvaginal cervical length measurement for prediction of preterm birth in women with threatened preterm labor: a meta-analysis. Ultrasound in Obstetrics, and Gynecology. 2010;35:54-64.

8. American College of Obstetricians, and Gynecologists. ACOG Practice Bulletin. Assessment of risk factors for preterm birth. Clinical management guidelines for obstetrician-gynecologists. Number 31 , October 2001. (Replaces Technical Bulletin number 206, June 1995; Committee Opinion number 172, May 1996; Committee Opinion number 187, September 1997; Committee Opinion number 198, February 1998; andCommittee Opinion number 251, January 2001). Obstetrics, and Gynecology. 2001;98:709-716.

9. Hoffmann JJ. Red cell distribution width, andmortality risk. Clin Chim Acta. 2012;413(7-8):824-825.

10. Christensen RD, Del Vecchio A, Henry E. Expected erythrocyte, platelet, andneutrophil values for term, andpreterm neonates. J Matern Fetal Neonatal Med. 2012;25(Suppl 5):77-79.

11. Park Y, Schoene N, Harris W. Mean platelet volume as an indicator of platelet activation: methodological issues. Platelets. 2002;13(5-6): 301-306.

12. Gasparyan AY, Ayvazyan L, Mikhailidis DP, et al. Mean platelet volume: a link between thrombosis, andinflammation?. Curr Pharm Des. 2011;17(1):47-58.

13. To MS, Skentou C, Chan $\mathrm{C}$, et al. Cervical assessment at the routine 23-week scan: st, andardizing techniques. Ultrasound in Obstetrics, and Gynecology. 2001;17:217-219.

14. Slattery MM, Morrison JJ. Preterm delivery. Lancet. 2002;360:14891497.

15. Gramellini D, Fieni S, Molina E, et al. Transvaginal sonographic cervical length changes during normal pregnancy. Journal of Ultrasound in Medicine. 2002;21:227-232.

16. Kiefer DG, Vintzileos AM. The utility of fetal fibronectin in the prediction, andprevention of spontaneous preterm birth. Reviews in Obstetrics, and Gynecology. 2008;1:106-112.
17. Goldenberg RL, Hauth JC, Andrews WW. Intrauterine infection, andpreterm delivery. The New Engl, and Journal of Medicine. 2000;342:1500.

18. Yoon BH, Yang SH, Jun JK, et al. Maternal blood C-reactive protein, white blood cell count, andtemperature in preterm labor: a comparison with amniotic fluid white blood cell count. Obstetrics and Gynecology. 1996;87:231-237.

19. Tzur T, Weintraub AY, Sergienko R, et al. Can leukocyte count during the first trimester of pregnancy predict later gestational complications? Archives of Gynecology, and Obstetrics. 2013;287:421-427.

20. Kim MA, Lee BS, Park YW, et al. Serum markers for prediction of spontaneous preterm delivery in preterm labor. European Journal of Clinical Investigation. 2011;41:773-780.

21. Ting HS, Chin PS, Yeo GS, et al. Comparison of bedside test kits for prediction of preterm delivery: phosphorylated insulin-like growth factor binding protein-1 (pIGFBP-1) test, andfetal fibronectin test. Annals of the Academy of Medicine. 2007;36:399-402.

22. Francesca G, Laura C, Iol anda M, et al. The red cell distribution width (RDW): Value, androle in preterm, IUGR (intrauterine growth restricted), full-term infants. Hematology. 2013;19:365-369.

23. Hatice KÖ, Faruk K, Sefa Ö, et al. The predictive value of red cell distribution width levels on mortality in intensive care patients with community-acquired intra-abdominal sepsis. Ulus Travma Acil Cerrahi Derg. 2015;21(5).

24. Erez O, Romero R, Hoppensteadt D, et al. Premature labor: a state of platelet activation? J Perinat Med. 2008;36(5):377-387.

25. Saleh AA, Bottoms SF, Farag AM, et al. Markers for endothelial injury, clotting, andplatelet activation in preeclampsia. Arch Gynecol Obstet. 1992;251:105-110.

26. Henn V, Slupsky JR, Grafe M, et al. CD40 lig, and on activated platelets triggers an inflammatory reaction of endothelial cells. Nature. 1998;391:591-594.

27. Ahmed MN, Hussein MA. Changes in platelet indices during pregnancy as potential markers for prediction of preeclampsia development. Open Journal of Obstetrics, and Gynecology. 2015;5:703-712.

28. Myatt L, Clifton RG, Roberts JM, et al. Eunice kennedy shriver national institute of child health, and human development (NICHD) maternalfetal medicine units (MFMU) network. First-trimester prediction of preeclampsia in nulliparouswomen at low risk. Obstet Gynecol. 2012;119:1234-1242.

29. Aynıoglu O, Isık H, Sahbaz A, et al. Can plateletcrit be a marker for recurrent pregnancy loss? Clin Appl Thromb Hemost. 2016;22(5):447452

30. Sahbaz A, Cicekler H, Aynioglu O, et al. Comparison of the predictive value of plateletcrit with various other blood parameters in gestational diabetes development. J Obstet Gynaecol. 2016;36(5):589-593.

31. Ekin A, Gezer C, Kulhan G, et al. Can platelet count, andmean platelet volume during the first trimester of pregnancy predict preterm premature rupture of membranes? J Obstet Gynaecol Res. 2015;41(1):23-28.

32. Gioia S, Piazze J, Anceschi MM, et al. Mean platelet volume: association with adverse neonatal outcome. Platelets. 2007;18(4):284-288. 